\title{
Malnutrition in the Outcome of Wound Healing at Public Hospitals in Bahir Dar City, Northwest Ethiopia: A Prospective Cohort Study
}

\author{
Netsanet Fentahun $\mathbb{D D}^{1}{ }^{1}$ Yeabsira Anteneh, ${ }^{2}$ and Yonatan Menber ${ }^{1}$ \\ ${ }^{1}$ Department of Nutrition and Dietetics, School of Public Health, College of Medicine and Health Science, Bahir Dar University, \\ Bahir Dar, Ethiopia \\ ${ }^{2}$ Felege Hiwot Comprehensive Specialized Hospital, Bahir Dar, Ethiopia
}

Correspondence should be addressed to Netsanet Fentahun; netsanetfentahun01@gmail.com

Received 12 September 2020; Revised 31 January 2021; Accepted 8 February 2021; Published 19 February 2021

Academic Editor: Elsa Lamy

Copyright (c) 2021 Netsanet Fentahun et al. This is an open access article distributed under the Creative Commons Attribution License, which permits unrestricted use, distribution, and reproduction in any medium, provided the original work is properly cited.

\begin{abstract}
Background. Poor nutritional status affects the normal process of the wound healing stage. There is limited evidence regarding the association between malnutrition and wound healing in Ethiopia. Objective. To assess the association between nutritional status and wound healing progress among adult individuals who had undergone abdominal surgery at Public Hospitals, Ethiopia. Methods. A prospective cohort study was conducted on 310 adult patients who had undergone abdominal surgery from August to December 2019. Data were collected using a standardized, structured, and pretested questionnaire. Anthropometric and serum albumin measurements were used to measure nutritional status. A multivariable Cox-regression analyses model was fitted to show the association between malnutrition and wound healing and $p$ value $<0.05$ was used to declare statistical significance value. Results. The cumulative incidence rate of good wound healing was 65.5\% (95\% CI: 60.0-71.0). Patients who had normal preoperative body mass index (adjusted hazard ratio $(\mathrm{AHR})=2.22$ (95\% CI: 1.55-3.19)) and normal range of serum albumin level $(\geq 3.5)(\mathrm{AHR}=1.56$ (95\% CI: 1.05-2.29)) were significantly associated with better wound healing outcomes. Conclusion. Nutritional status had a strong association with good wound healing outcomes. Therefore, nutritional status screening should be done for all adult patients before undergoing abdominal surgery to improve wound healing outcomes and reduce hospital stays.
\end{abstract}

\section{Background}

The wound is defined as damage, disruption, or loss of the normal anatomical structure and functional continuity of living tissues accidentally, intentionally, or as a result of a disease process [1]. Wound healing is a continuing and complex process of changing injured tissue to another new tissue with the coordination of several biological and molecular events, which directly correlated to patients' overall nutritional status [2].

Poor nutritional status affects the normal process of the wound healing stage by prolonging the inflammatory phase, decreasing fibroblast proliferation, and altering collagen production which decreases wound tensile strength and increased infection rates $[3,4]$. Previous studies found that wound is a common surgical problem all over the world that causes significant morbidity and mortality. Approximately 3-6 million people are affected by nonhealing wounds in the United States of America [5-7]. The incidence rate of the postoperative complicated outcome was $4-7 \%$ in Canada, $10.33 \%$ in Barcelona city, $38.8 \%$ in Sub-Saharan Africa, and $19.1 \%$ in Ethiopia from admitted surgical patients [8-11].

The patient with hypoalbuminemia who had undergone abdominoperineal resections was more likely to increase the risk of delayed wound healing as compared with the wellnourished [12]. While on serum albumin measurement operated surgical patients with normal nutritional status had a higher probability of wound healing than the counterpart [13]. The percentage of postoperative wound healing incidence rate was $11.40 \%$ from the healthy individuals but 
$2.30 \%$ in malnourished patients [14]. Based on serum albumin measure, the incidence rate of delayed wound healing was $12 \%$ and $28 \%$ from the well-nourished and undernourished, respectively. Based on body mass index (BMI) measure, the incidence rate of delayed wound healing was $12 \%$ and $21 \%$ from the well-nourished and undernourished, respectively [15].

Even though the postoperative complication rate of abdominal surgery patients in the Sub-Saharan African setting is still high [16], there is limited published evidence on the incidence of wound healing associated with the effect of nutritional status among patients undergoing abdominal surgery in Sub-Saharan African especially in Ethiopia. Also, there are no nutrition screening services in Ethiopia before undergoing abdominal surgery. This makes the outcome of abdominal surgery the worst and a long hospital in Ethiopia. Therefore, this study will be important to generate evidence on the association between malnutrition and wound healing progress among adult patients undergoing abdominal surgery in Ethiopia. Likewise, this study helps to change the usual trend of the integration of nutrition screening services with clinical management.

\section{Methods}

2.1. Study Design and Setting. Prospective cohort study design was conducted at Bahir Dar City Public Hospitals in Amhara Region, Ethiopia, from August to December 2019. Felege Hiwot Comprehensive Specialized Hospital (FHCSH), Tibebe Ghion Specialized Hospital (TGSH), and Addis Alem Primary Hospital (AAPH) were included in the study.

\subsection{Study Population, Sample Size, and Sampling Procedure.} The study population was adult patients ( $\geq 18$ years) who underwent abdominal surgery at the surgical ward of Bahir Dar City Public hospitals. The sample size was calculated by considering $95 \%$ confidence level (CL), $80 \%$ of power $(1-\beta)$, and taking nutritional status with1:1 ratio (well-nourished vs. malnourished) as exposure variable [14] and 10\% nonresponse rate. The final sample size was 310 adult patients (155 for well-nourished and 155 malnourished patients) who had undergone abdominal surgery.

Three hundred ten adult abdominal surgical operation patients were selected by using a systematic sampling technique. The final sample size was proportionally allocated to the selected hospitals based on their last four-month abdominal surgical operation reports. The first case was selected by lottery method and then every three cases were included for both well-nourished and malnourished patients.

\subsection{Data Collection Procedure and Quality Improvement.} Data were collected using a standardized, structured, and face-to-face interviewer-administered questionnaire. The questionnaire includes the patient's sociodemographic characteristics, clinical features of patients, lifestyle and behavior of patients, and nutritional status which include the anthropometric examination and biochemical test. Data were collected by six trained B.Sc., Nurse and three trained medical laboratory technology with fluency in the local language (Amharic). Orientation, supervision, and reviewing the completed questionnaire were done to assure data quality. The data collectors measured the patient's preoperative nutritional status (BMI and serum albumin level) and followed postoperative wound healing progress with clinical features.

2.4. Anthropometric Measurements. Body weight was measured twice to the nearest $0.1 \mathrm{~kg}$, and the average reading was calculated. While measuring the body weight, each patient was asked to take off the heavy clothes and shoes to keep in light clothes and remove heavy objects such as wallet and mobile before standing on the calibrated weighing scale balance (SECA, Germany) before the operation.

Height was measured twice by using SECA calibrated Stadiometer read as in centimeter. Each individual was asked to stand as fully erected as possible as against the wall and maintain the head in a position of straight-forward gaze and feet slightly apart with the back of the head, scapulae, buttocks, and heels positioned in contact with the wall; then, the sliding bar was lowered horizontally to the participants head to where the bar was locked and the measurement read and recorded during admission. Well-nourished patients' BMI were between 18.5 and $24.9 \mathrm{~kg} / \mathrm{m}^{2}$, while malnourished patients' BMI were ${ }^{<} 18.5 \mathrm{~kg} / \mathrm{m}^{2}$ (underweight) or $\geq 25 \mathrm{~kg} / \mathrm{m}^{2}$ (overweight) [17].

2.5. Biochemical Test. Blood specimens were taken from sample patients before undergoing abdominal surgery. The medical laboratory technologists analyzed the serum albumin level as per the standard operating procedure. And then serum albumin level was classified as the normal range $(>3.5 \mathrm{~g} / \mathrm{dl})$ and not the normal range $(<3.5 \mathrm{~g} / \mathrm{dl})$ [17].

2.6. Wound Healing Follow-Up Checklist. All study patients were followed-up and their wound healing characteristics were assessed throughout the $1^{\text {st }}, 3^{\text {rd }}, 5^{\text {th }}, 7^{\text {th }}$, and $10^{\text {th }}$ postoperative day. The data collectors estimated and recorded the wound healing progress by assessing sign and symptom of wound healing character (bleeding, scab formation, localized pain and swelling, persistent fever, red streaks near the cut, fouler odor, darkening skin edges, clear fluid, and thick grayish discharge around incision site) within ten postoperative days based on wound healing checklist. And also the level of the surgeon, regular wound care provider, patient's medication, privies surgical history, chronic comorbidity, mode, and type of surgery was assessed.

2.6.1. Good Wound Healing. It includes patients who showed improvement regarding any sign of bleeding, swelling, localized pain, fever, redness, grayish or clear fluid discharge, and fouler odor, with the incision site filled by 
repaired tissue with the sign of shiny and smooth scar formation within ten postoperative days.

2.6.2. Poor Wound Healing. It includes patients who had any sign of bleeding, persistent fever, red streaks near the cut, increasing localized pain and swelling, fouler odor, darkening skin edges, and thick-grayish or clear fluid discharge from the incision site until the $10^{\text {th }}$ postoperative days [18]. Finally, the patients who had good wound healing outcomes within ten postoperative days are considered as an event, and patients who had poor wound healing progress and were lost to follow-up (died, medical against, referral, defaulter, and self-discharge) until the $10^{\text {th }}$ postoperative day was considered as censored.

2.7. Statistical Analysis. Data were checked manually for completeness and consistencies and then coded and entered into EPI Data version 3.1 software and exported to SPSS (statistical package for social science) version 23 software package for further analysis. Descriptive statistics were used to describe variables and summarize the data in the form of frequency, mean, standard deviation, cross-tabulation, table, and graph.

Multivariable Cox-regressions analysis models were used to determine the association between malnutrition and wound healing outcomes. The model assumption was checked by using $\log (-\log ) \mathrm{S}(\mathrm{t})$ plots. Crude and adjusted hazard ratios with their $95 \%$ confidence interval (CI) were estimated, and $P$ value less than 0.05 was considered as the significance level for associations between dependent and predictor variables.

\section{Results}

3.1. Sociodemographic Characteristics of Participants. A total of 310 patients had undergone abdominal surgery at Bahir Dar City Public Hospitals in Amhara Region. The mean ages of the patients were $39.7 \pm 16.66$ years $( \pm S D)$. The majority $(92.3 \%)$ of patients were Orthodox Christianity followers. More than half $(59.4 \%)$ of the patients were from urban residency. 191 (61.6\%) patients were married, and higher proportions (33.5\%) of patients had attended primary education. More than half $(57.4 \%)$ of patients were recruited from FHCSH (Table 1).

3.2. Patients 'Lifestyle. 21 (6.8\%) patients smoke cigarettes, and $96(31.0 \%)$ patients were drinking different types of alcohol. 178 (57.4\%) were walking from home to the workplace on foot. 138 (44.5\%) had handwashing practice before eating their meals, while 281 (90.6\%) patients sometimes had handwashing practice after toilet. $188(60.6 \%)$ patients used pipe water for drinking purposes. 14 patients (4.5\%) were doing regular physical exercises (Table 2 ).

3.3. Nutritional Status of Patients. According to BMI measurement, $155(50.0 \%)$ preoperative patients were malnourished (48.4\% underweight and $1.6 \%$ overweight).
According to serum albumin level measurement, 171 (55.2\%) preoperative patients were well-nourished.

3.4. Clinical Feature of Patients. 159 (51.3\%) patients were carried out with an emergency operation table. The most frequent type of abdominal surgery was laparotomy (39.4\%). $242(78.1 \%)$ were operated on by a senior physician. 148 (47.7\%) patients have developed a disease process before two weeks, whereas $39(12.6 \%)$ patients had a history of chronic comorbidity (Table 3 ).

3.5. Outcomes of Wound Healing. The overall incidence rate of good wound healing was $65.5 \%$ (95\% CI: 60.0-71.0). From normal preoperative BMI patients, $88.4 \%$ had a good wound healing incidence rate whereas malnourished preoperative patients, $42.6 \%$, had a good wound healing incidence rate. Likewise, from well-nourished preoperative patients in serum albumin measurement, $86.0 \%$ had good wound healing outcomes. Preoperative malnourished patients in serum albumin measurement had $40.3 \%$ of good wound healing outcomes.

3.6. Malnutrition and Wound Healing Outcomes. Before fitting the covariate into the model, the proportional hazard assumption was checked using Log (-Log) S (t) plots. The final model was adjusted with the type of medication, type of surgery, acute illness, chronic comorbidity, smoking, age of patients, and sex to know the association between malnutrition and wound healing outcomes. In the multivariable Cox regression analysis model, BMI and serum albumin test level were significant predictors of wound healing among adult patients who underwent abdominal surgery.

Patients who had the normal preoperative body mass index were 2 times more likely to have good wound healing incidence rate outcomes as compared to those malnourished patients ( $\mathrm{AHR}=2.22$ (95\% CI: $1.55-3.19))$. Patients who had a normal range of serum albumin level $(>3.5)$ were 1.6 times more likely to have good wound healing status as compared to malnourished patients $(\mathrm{AHR}=1.56(95 \% \mathrm{CI}: 1.05-2.29))$ (Table 4).

\section{Discussion}

Wound healing is a complex and dynamic process that needs well-nutritional status for the rapid inflammatory phase, promote collagen production, enhance fibroblast proliferation, and fast granulation to increase wound tensile strength for good wound healing outcome. Preoperative well-nutritional status is a fundamental aspect for optimal wound healing which decreases hospital length of the stay, minimizes health care cost, and reduces postoperative infection and complication rate [19]. As the finding of this study, out of 310 adult patients who had undergone an abdominal surgical procedure, 209 patients were improved from surgical incision which gives $65.0 \%$ with $95 \% \mathrm{CI}$ : $0.60-0.71$ of overall good wound healing incidence rate. The finding was similar to studies done in India 63\% [15], Brazil 
TABLE 1: Sociodemographic characteristics of adult patients who had undergone abdominal surgery at Bahir Dar City Public Hospitals, Northwest Ethiopia, $2019(N=310)$.

\begin{tabular}{|c|c|c|c|}
\hline Sociodemographic character & Categories & Frequency & Percentage \\
\hline \multirow{3}{*}{ Study settings } & FHCSH & 178 & 57.4 \\
\hline & TGSH & 88 & 28.4 \\
\hline & AAPH & 44 & 14.2 \\
\hline \multirow{3}{*}{ Age in year } & $18-40$ & 173 & 55.8 \\
\hline & $41-65$ & 93 & 30.0 \\
\hline & $>65$ & 44 & 14.2 \\
\hline \multirow{2}{*}{ Sex } & Male & 184 & 59.4 \\
\hline & Female & 126 & 40.6 \\
\hline \multirow{2}{*}{ Religion } & Orthodox & 286 & 92.3 \\
\hline & Others $^{\mathrm{a}}$ & 24 & 7.7 \\
\hline \multirow{4}{*}{ Marital status } & Married & 191 & 61.6 \\
\hline & Single & 82 & 26.5 \\
\hline & Divorced & 20 & 6.5 \\
\hline & Widowed & 17 & 5.5 \\
\hline \multirow{4}{*}{ Education } & No formal education & 50 & 16.1 \\
\hline & Primary & 104 & 33.5 \\
\hline & Secondary & 75 & 24.2 \\
\hline & More than secondary & 81 & 26.1 \\
\hline \multirow{5}{*}{ Occupation } & Farmer & 98 & 31.6 \\
\hline & Merchant & 75 & 24.2 \\
\hline & Employed & 64 & 20.6 \\
\hline & Student & 29 & 9.4 \\
\hline & Others ${ }^{\mathrm{b}}$ & 25 & 8.1 \\
\hline \multirow{2}{*}{ Residency } & Urban & 184 & 59.4 \\
\hline & Rural & 126 & 40.6 \\
\hline
\end{tabular}

${ }^{a}$ Muslim and protestant, ${ }^{\mathrm{b}}$ daily labor, retired, housewife; FHCSH: Felege Hiwot Comprehensive Specialized Hospital; TGSH: Tibebe Ghion Specialized Hospital; AAPH: Addis Alem Primary Hospital.

TABle 2: Lifestyle of adult patients undergoing abdominal surgery at Bahir Dar City Public Hospitals, Northwest Ethiopia, 2019 (N=310).

\begin{tabular}{|c|c|c|c|}
\hline Variables & Categories & Frequency & Percentage \\
\hline \multirow{2}{*}{ Cigarette smoking } & Yes & 21 & 6.8 \\
\hline & No & 289 & 93.2 \\
\hline \multirow{2}{*}{ Duration of smoking $(n=21)$} & Daily & 8 & 2.6 \\
\hline & Occasionally & 13 & 4.2 \\
\hline \multirow{2}{*}{ Alcohol consumption } & Yes & 96 & 31.0 \\
\hline & No & 214 & 69.0 \\
\hline \multirow{2}{*}{ Frequency of alcohol consumption per day $(n=96)$} & $\leq$ three times a day & 62 & 20.0 \\
\hline & $>$ three times a day & 34 & 11.0 \\
\hline \multirow{2}{*}{ Regular physical exercise } & Yes & 14 & 4.5 \\
\hline & No & 296 & 95.5 \\
\hline \multirow{2}{*}{ Duration of physical exercise $(n=14)$} & $\leq$ three times a week & 14 & 4.5 \\
\hline & $>$ three times a week & 0 & 0.0 \\
\hline \multirow{2}{*}{ Means of transport from home to workplace and workplace to home } & On foot & 178 & 57.4 \\
\hline & On transportion & 132 & 42.6 \\
\hline \multirow{3}{*}{ Source of drinking water } & Pipe water & 188 & 60.6 \\
\hline & Springwater & 98 & 31.6 \\
\hline & Well water & 24 & 7.7 \\
\hline \multirow{2}{*}{ Handwashing after toilet } & Always & 29 & 9.4 \\
\hline & Sometimes & 281 & 90.6 \\
\hline \multirow{2}{*}{ Handwashing before eating } & Always & 138 & 44.5 \\
\hline & Sometimes & 172 & 55.5 \\
\hline
\end{tabular}


TABLE 3: Clinical features of adult patients who had undergone abdominal surgery at Bahir Dar City Public Hospitals, Northwest Ethiopia, $2019(N=310)$.

\begin{tabular}{|c|c|c|c|}
\hline Variables & Categories & Frequency & Percentage (\%) \\
\hline \multirow{5}{*}{ Type of current surgery } & Laparotomy & 122 & 39.4 \\
\hline & Herniorrhaphy & 60 & 19.4 \\
\hline & Appendectomy & 73 & 23.5 \\
\hline & Cholecystectomy & 36 & 11.6 \\
\hline & Others & 19 & 6.1 \\
\hline \multirow{3}{*}{ Level of an intervening surgeon } & IESO & 21 & 6.8 \\
\hline & Resident & 47 & 15.2 \\
\hline & Senior & 242 & 78.1 \\
\hline \multirow{2}{*}{ Mode of surgery } & Elective & 151 & 48.7 \\
\hline & Emergency & 159 & 51.3 \\
\hline \multirow{2}{*}{ Duration of the disease process } & $<$ Two weeks & 162 & 52.3 \\
\hline & $\geq$ Two weeks & 148 & 47.7 \\
\hline \multirow{2}{*}{ Acute illness in the last 1 month } & Yes & 115 & 37.1 \\
\hline & No & 195 & 62.9 \\
\hline \multirow{2}{*}{ History of chronic comorbidities } & Yes & 39 & 12.6 \\
\hline & No & 271 & 87.4 \\
\hline \multirow{2}{*}{ Type of chronic comorbidities } & Malignancy & 17 & 5.5 \\
\hline & Others $^{\mathrm{h}}$ & 22 & 7.1 \\
\hline \multirow{2}{*}{ History of surgical intervention } & Yes & 8 & 2.6 \\
\hline & No & 202 & 97.4 \\
\hline \multirow{3}{*}{ Type of patients current medication } & NSAIDS & 62 & 20.0 \\
\hline & Antibiotic & 22 & 7.1 \\
\hline & Both & 226 & 72.9 \\
\hline
\end{tabular}

${ }^{{ }^{E} \text { Excision and achalasia, }}{ }^{\mathrm{h}}$ tuberculosis and diabetic mellitus, HIV/ADIS, IESO: Integrated Emergency Surgical Officers.

TABle 4: Predictors of wound healing among adult patients undergoing abdominal surgery at Bahir Dar City Public Hospitals, Northwest Ethiopia, $2019(N=310)$.

\begin{tabular}{lcccc}
\hline Variables & & & Wound healing status \\
& Good (\%) & Censored (\%) & CHR (95\%CI) & AHR (95\% CI) \\
\hline $\begin{array}{l}\text { Body Mass index\# } \\
\text { Malnourished }\end{array}$ & $66(42.6)$ & $89(57.4)$ & 1 & 1 \\
Well-nourished & $137(88.4)$ & $18(11.6)$ & $3.18(2.37-4.29)$ & $2.22(1.55-3.19)^{* * *}$ \\
\hline $\begin{array}{l}\text { Serum albumin\# } \\
<3.5\end{array}$ & $56(40.3)$ & $83(59.7)$ & 1 & 1 \\
$\geq 3.5$ & $147(86.0)$ & $24(14.0)$ & $3.16(2.31-4.30)$ & $1.56(1.05-2.29)^{*}$ \\
\hline
\end{tabular}

\#: adjusted with the type of medication, type of surgery, acute illness, chronic comorbidity, smoking, age of patients, and sex; ${ }^{*}=P$ value $<0.05$ and ${ }^{* * * *}=P$ value $\leq 0.001$; CHR: crude hazard ratio; AHR: adjusted hazard ratio.

$67.8 \%$ [13], and Gaza Strip 65\% [20], but lower compared to studies done in Korea $73 \%$ [21] and Italy 90\% [22], the discrepancy might be due to difference of socioeconomic status, the magnitude of sample size, distribution of geographical area, and different sit of the incisional wound and mode of surgery (only elective or emergency or both elective and emergency surgery).

In this study nutritional status was found to be significantly associated with wound healing outcomes. This result was in agreement with other studies done in India [14], Italy [22], India [15], and Korea [21]. This finding could be explained by the fact that the patients had a good nutritional status which enhances the growth hormone and collagen production that increase tensile strength and decrease risk factor of wound healing dalliance.
While observing the actual practice in Ethiopian health facilities, there are no nutrition screening services before undergoing abdominal surgery. This makes the outcome of abdominal surgery worse and a long time hospitalization hospital. This study shows nutrition screening before undergoing abdominal surgery is an important point to improve wound healing outcomes and shorting hospitalization.

This study does not consider the effect of postoperative hospital-acquired malnutrition during a hospital stay because of the difficulty to measure the postoperative nutritional status of patients. This current follow-up study did not follow up those patients once who had good wound healing outcomes and discharge even if the wound relapses and develops any sign of poor wound healing at home. In this study, the patient's dietary intake habit and immunity do not 
include a predicted variable due to difficulty to measure. Despite limitations, this study provides vital baseline information on incidence rate and predictors of wound healing among adult patients who had undergone abdominal surgery at Bahir Dar City Public Hospitals, Northwest Ethiopia.

\section{Conclusion}

Nutritional status had a strong association with good wound healing outcomes. Therefore, nutritional status screening should be done for all adult patients before undergoing abdominal surgery to improve wound healing outcomes and reduce hospital stays.

\section{Abbreviations}

AAPH: Addis Alem Primary Hospital

AHR: Adjusted hazard ratio

BMI: $\quad$ Body mass index

CL: $\quad$ Confidence level

CHR: Crude hazard ratio

DM: $\quad$ Diabetes mellitus

FHCSH: Felege Hiwot Comprehensive Specialized Hospital

TGSH: Tibebe Ghion Specialized Hospital

WHO: World Health Organization.

\section{Data Availability}

The datasets supporting the conclusions of this article are included in the article.

\section{Ethical Approval}

Ethical clearance was obtained from the Institutional Review Board, College of Medicine and Health Sciences, Bahir Dar University, Ethiopia. Permission letters were obtained from each Bahir Dar City Public Hospital's manager.

\section{Consent}

Written consent was obtained from the patient after providing information about the purpose of the study.

\section{Conflicts of Interest}

The authors declare that they have no conflicts of interest.

\section{Authors' Contributions}

NF, YA, and YM assisted in the conception of the study; developed the proposal; were involved in the method development, analysis, interpretation of the findings, and development of the manuscript. All the authors read, revised, and approved the final manuscript.

\section{Acknowledgments}

The authors would like to thank Bahir Dar University, data collectors, supervisors, and study participants.

\section{References}

[1] M. C. D. L. S. Robson and M. G. Franz, "Wound healing: biologic features and approaches to maximize healing trajectories," Current Problems in Surgery, vol. 2, no. 38, pp. 72-140, 2001.

[2] A. C. A. K. Campos and A. B. Branco, "Assessment and nutritional aspects of wound healing," Current Opinion in Clinical Nutrition \& Metabolic Care, vol. 11, no. 3, pp. 281288, 2008.

[3] J. K. Stechmiller, "Understanding the role of nutrition and wound healing," Nutrition in Clinical Practice, vol. 25, no. 1, pp. 61-68, 2010.

[4] B. A. S. Witts and T. Martin, "The role of nutrition in successful wound healing," Journal of Community Nursing, vol. 32, no. 4, 2018.

[5] N. Menke, R. W. Kevin, T. Witten, and D. Bonchev, "Impaired wound healing," Clinics in Dermatology, vol. 25, no. 1, pp. 19-25, 2007.

[6] K. Järbrink, G. Ni, H. Sönnergren et al., "Prevalence and incidence of chronic wounds and related complications: a protocol for a systematic review," Systematic Reviews, vol. 5, no. 1, p. 152, 2016.

[7] F. Gottrup, "A specialized wound-healing center concept: the importance of a multidisciplinary department structure and surgical treatment facilities in the treatment of chronic wounds," The American Journal of Surgery, vol. 187, no. 5, pp. S38-S43, 2004.

[8] K. Denny, C. Lawand, and S. Perry, "Compromised wounds in Canada," Healthcare Quarterly Toronto, Ont, vol. 17, no. 1, pp. 7-10, 2013.

[9] A. Lanau-Roig, "Time of Chronic wound healing, as part of a prevalence and incidence study," Enfermería Global, vol. 16, no. 2, pp. 445-453, 2017.

[10] T. L. Laloto, D. H. Gemeda, and S. H. Abdella, "Incidence and predictors of surgical site infection in Ethiopia: a prospective cohort," BMC Infectious Diseases, vol. 17, no. 1, p. 119, 2017.

[11] C. G. M. Tebou, N. T. Maxou, A. Esiene, B. O. Nana, J. N. Jean, and E. Sobngwi, "Impact of perioperative nutritional status on the outcome of abdominal surgery in a sub-Saharan Africa setting," BMC Research Notes, vol. 10, no. 1, p. 484, 2017.

[12] C. C. Chang, Y.-T. Lan, J.-K. Jiang et al., "Risk factors for delayed perineal wound healing and its impact on prolonged hospital stay after abdominoperineal resection," World Journal of Surgical Oncology, vol. 17, no. 1, p. 226, 2019.

[13] E. L. Borges, J. F. P. Júnior, M. N. S. Abreu, V. L. de Araújo Lima, P. A. B. Silva, and S. M. Soares, "Factors associated with the healing of complex surgical wounds in the breast and abdomen: retrospective cohort study," Revista latino-americana de enfermagem, vol. 24, 2016.

[14] R. Singh, H. S. Tripathi, A. Tripathi, and P. Himansha, "Assessment of wound healing in relation to nutritional status of the patients in common surgical condition in tertiary care centre," International Surgery Journal, vol. 6, no. 4, pp. 1247-1253, 2019.

[15] C. C. Mahakalkar, M. Suchita, Y. Meenakshi et al., "Malnutrition in hospitalised patients; a real concern in surgical outcomes," International Journal of Research in Medical Sciences, vol. 2, no. 1, pp. 250-257, 2014.

[16] T. Uribe-Leitz, J. J. Maurer, M. M. Esquivel, A. A. Gawande et al., "Variability in mortality following cesarean delivery, appendectomy, and groin hernia repair in low-income and middle-income countries. a systematic review and analysis of 
published data," Lancet Glob Health, vol. 4, no. 3, pp. e165e74, 2016.

[17] WHO, Global Database on Body Mass Index, WHO, Geneva, Switzerland, 2016.

[18] G. Gordillo, Six Signs Your Wound Is Not Healing Right, The Ohio State University, Columbus, OH, USA, 2017.

[19] P. Beldon, "Basic science of wound healing," Surgery, Oxford, vol. 28, no. 9, pp. 409-412, 2010.

[20] S. M. Hammad, I. A. Naser, H. T. Mahmmoud, and S. A. Ayman, "Dietary intake and biochemical indicators and their association with wound healing process among adult burned patients in the gaza strip," Current Research in $\mathrm{Nu}$ trition and Food Science, vol. 7, no. 1, 2019.

[21] J.-G. K. Seung-Jin Kwag, K. Won-Kyung, L. Jin-Kwon, and O. Seong-Taek, "The nutritional risk is an independent factor for postoperative morbidity in surgery for colorectal cancer," Annals of Surgical Treatment and Research, vol. 86, no. 4, 2014.

[22] V. Mignini, E. Lattanz, V. Valeri, N. Clementi, A. Gasbarrini, and C. Rasett, "Impact of patients nutritional status on major surgery outcome," European Review for Medical and Pharmacological Sciences, vol. 22, pp. 3524-3533, 2018. 